\title{
Equivalence of $\mathrm{BCSH}$ and $\mathrm{WHO}$ diagnostic criteria for ET
}

CN Harrison, MF McMullin, AR Green and AJ Mead

Leukemia (2017) 31, 1660; doi:10.1038/leu.2017.119; published online 5 May 2017

Correction to: Leukemia (2017) 31, 527-528; doi:10.1038/ leu.2016.318; published online 29 November 2016

Following the publication of this article, the authors noted that the following acknowledgement needed to be included:
'AM acknowledges funding from The Haematology and Stem Cell Theme of the Oxford Biomedical Research Centre (BRC).' 